\title{
Mid-term results show no significant difference in postoperative clinical outcome, pain and range of motion between a well-established total knee arthroplasty design and its successor: a prospective, randomized, controlled trial
}

\author{
Georg Hauer ${ }^{1}$. Nina Hörlesberger ${ }^{1}$. Sebastian Klim ${ }^{1}$. Gerwin A. Bernhardt ${ }^{1}$. Lukas Leitner ${ }^{1} \cdot$ Mathias Glehr $^{1}$. \\ Andreas Leithner ${ }^{1} \cdot$ Patrick Sadoghi ${ }^{1}$ (I)
}

Received: 14 February 2020 / Accepted: 21 April 2020 / Published online: 24 April 2020

(c) The Author(s) 2020

\begin{abstract}
Purpose The purpose of this study was to compare the clinical and functional outcome scores following total knee arthroplasty (TKA) with two different systems. The hypothesis was that there is a difference between patients receiving the newer design than those receiving the predecessor.

Methods Two hundred patients who underwent TKA were randomized into two groups: patients received either Attune TKA or PFC Sigma (both DePuy Synthes, Warsaw, IN). Clinically, the Knee Society Knee and Function Scores (KS and FS), Western Ontario and McMaster Universities Osteoarthritis Index (WOMAC), Range of Motion (ROM) and Visual Analogue Scale (VAS) were evaluated and compared between the groups 2 years after surgery. 158 patients (80 in the Attune group and 78 in the PFC Sigma group) were available for follow-up.

Results Through bivariate analysis using parametric and non-parametric statistical tests, no significant differences in postoperative KS, FS, WOMAC, ROM or VAS between the two groups were detected. Both groups significantly improved regarding all evaluated endpoints 2 years after surgery.

Conclusions In the current study population, no difference in clinical outcome between the two systems was found. The expected benefits of design modifications could not be observed in clinical outcome scores 2 years postoperatively. Both designs are effective options for improving pain and function in end-stage osteoarthritis.
\end{abstract}

Level of evidence I.

Keywords Total knee arthroplasty · Total knee replacement · TKA · Outcomes · Attune $\cdot$ PFC sigma

\section{Introduction}

Although TKA is a highly effective procedure, up to $20 \%$ of patients continue to suffer from pain after the replacement of the joint [5]. To address this difficulty, the number of implants available on the market has substantially increased, often with little or no evidence of clinical effectiveness or cost-effectiveness [5].

According to the national arthroplasty registry data, the Press Fit Condylar (PFC) Sigma (DePuy Synthes, Warsaw,

Patrick Sadoghi

patrick.sadoghi@medunigraz.at

1 Department of Orthopaedics and Trauma, Medical University of Graz, Auenbruggerplatz 5, 8036 Graz, Austria
IN) was one of the ten most used prostheses in primary TKA [1,9] worldwide. Despite very promising survival rates after long-term follow up, the PFC Sigma has continued to be associated with relatively high rates of dissatisfaction at short-to-midterm follow-ups [10]. In 2013, the company revealed a modification of the design. The Attune TKA System (DePuy Synthes, Warsaw, IN) was designed to address these rates by optimising the guidance in femoral size measurement and the flexion gap balancing using a single tool [11]. The new prosthesis was developed to more closely resemble the anatomical trochlear groove and patella as patellofemoral maltracking, tilt, and overstuffing have been shown to be important factors leading to pain and thus to unsatisfying results [4]. 
As the two TKA designs are amongst the most frequently used knee replacement systems worldwide, their clinical outcome is of great importance for orthopaedic surgeons $[1,9]$. The current literature suggests that both implants perform equally well, but well-designed, independent studies on the subject are required to clearly evaluate the effect of the implant design on the postoperative functional outcome $[6,8,10,12]$. This paper gives further insight into this evaluation as no prospective, randomized, controlled trial has yet been conducted to compare both designs. Therefore, the aim of this study was to compare the well-established PFC system and the Attune TKA using clinical outcome scores after 2-year follow-ups. The study's hypothesis was that modifications of the design of the Attune system would lead to an improved clinical outcome compared to the PFC Sigma system.

\section{Methods}

A prospective, randomized, parallel-group study was conducted in a single, urban, high-volume university hospital. Participants were recruited from the orthopaedic outpatient clinic. During their attendance patients were assessed both for their eligibility and their willingness to participate. Patients were eligible for inclusion in the trial if a decision had been made to have primary total knee arthroplasty surgery, but no particular type of prosthesis had been determined. All the patients were suffering from severe osteoarthritis of the knee confirmed by anteroposterior and lateral radiographs (Kellgren-Lawrence-Score III/IV) and high levels of pain in at least two knee compartments despite conservative treatment. Exclusion criteria were a flexion of less than $70^{\circ}(n=7)$, varus and valgus deformities of more than $15^{\circ}(n=11)$, patients with known or suspected metal allergies $(n=4)$ and patients under 60 years of age $(n=35)$. Patients who were unwilling to participate $(n=2)$ or unable to provide informed consent $(n=1)$ were also excluded.

Included patients were randomized and received either an Attune or a PFC Sigma TKA (both DePuy Synthes, Warsaw, IN). Participants were randomly assigned 1:1 the day before surgery using the Randomizer for Clinical Trials tool developed at the Medical University of Graz. A study nurse not involved in participant recruitment and patient care prepared the randomization in advance. Neither the patients nor the surgeons were blinded to the treatment allocation. The recruitment of study participants finished as soon as 100 patients were included in each group.

All procedures were carried-out by two senior knee surgeons using the same surgical technique with no patella resurfacing. Femoral and tibial components were cemented (Palacos R + G, Heraeus Medical, Wehrheim, Germany) and the tibia first method with the balancing of the flexion gap was carried out using a medial parapatellar approach. Postoperatively, patients followed a standardized rehabilitation protocol consisting of full weight bearing immediately after surgery with the use of crutches and continuous passive motion (CPM) therapy on the first postoperative day.

\section{Study population}

Out of two hundred consecutively enrolled patients, 80 patients in the Attune group and 78 patients in the PFC group were assessed at the 2-year follow-up analysis. A patient flow chart on study inclusion has been provided in Fig. 1. One patient in the Attune group and three patients in the PFC group had died of causes unrelated to their knee surgery. Twelve patients refused their further participation in the study at a final follow-up examination. Twenty-eight patients were lost to follow-up as they could not be contacted or did not appear on the scheduled examination dates. According to a systematic search of the patients' medical records, none of the patients lost to follow up or who refused their further participation had undergone revision surgery in the center hospital or regional partner hospitals. There were no significant differences in the demographic data between the two groups (Table 1).

\section{Outcome measurement}

The preoperative clinical status and results 2 year postoperatively were evaluated according to Knee Society Knee and Function Scores (KS and FS) [7], the Western Ontario and McMaster Universities Osteoarthritis Index (WOMAC) [3], the Visual Analogue Scale (VAS), and range of motion (ROM). The ROM was measured with a double-armed goniometer.

The study procedure followed accepted ethical, scientific, and medical standards and was conducted in compliance with recognized international standards, including the principles of the Declaration of Helsinki. Informed consent was obtained from all the study's participants and the study protocol was approved by the ethics committee of the Medical University of Graz (26-303 ex 13/14).

\section{Statistical analysis}

The data were analysed by SPSS Version 23.0 (IBM Corporation, New York, USA). Descriptive statistics for continuous variables were reported as the mean and standard deviation (SD). Categorical variables were reported as count and proportions. For comparisons of categorical variables, the chi-square exact test was used. The data were tested for normality using the Kolmogorov-Smirnov test, which revealed a parametric distribution for the BMI and a nonparametric distribution for all clinical scores and ROM. 


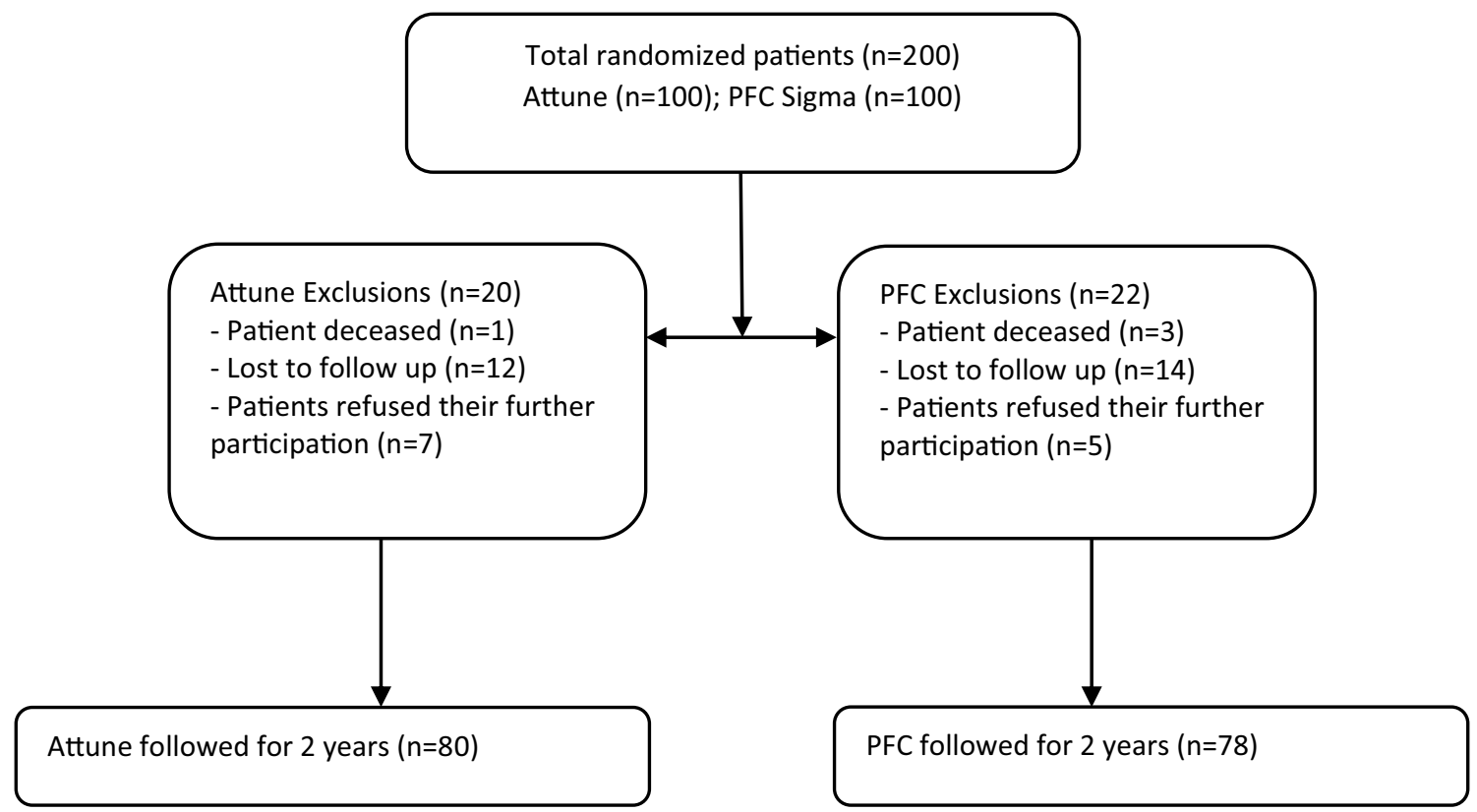

Fig. 1 Flow chart on study inclusion

Table 1 Patient demographics and baseline characteristics show no significant (n.s.) differences between our study groups

\begin{tabular}{llll}
\hline Demographics & Attune $(n=80)$ & PFC $(n=78)$ & $p$ value \\
\hline Sex $(\mathrm{M} / \mathrm{F})(n / \%)$ & $28(35 \%) / 52(65 \%)$ & $28(35.9 \%) / 50(64.1 \%)$ & n.s \\
Age $($ years), mean (SD) & $71.1(8.3)$ & $69.0(8.0)$ & n.s \\
BMI $\left(\mathrm{kg} / \mathrm{m}^{2}\right)$, mean (SD) & $28.8(4.4)$ & $29.4(4.6)$ & n.s \\
\hline
\end{tabular}

Differences between pre-operative and post-operative data were observed through the $t$ test and the Mann-Whitney $U$ test and the Wilcoxon signed-rank test. A $p$ value of $<0.05$ was interpreted as being statistically significant. An a priori power analysis revealed that a minimum of 64 patients per group would be necessary to detect a clinically relevant difference of $10^{\circ}$ in $\mathrm{ROM}$ with a SD of $20^{\circ}$ at a significance level of $p<0.05$ with a power of 0.8 . To compensate for dropouts, 100 study participants per group was considered a sufficient number.

\section{Results}

\section{Clinical results}

There were no significant differences in terms of pre- and postoperative KS and FS, WOMAC, VAS and ROM between the two groups (Table 2). At the 2-year follow-ups, statistically significant $(p<0.001)$ improvements were obtained in all clinical scores and ROM within the groups compared to preoperative values (Table 2). In the final follow-up cohorts, no minor or major complications that required further surgical treatment were observed.

\section{Discussion}

The most important finding of this study was that both systems provided excellent clinical results at the 2-year followups. However, no superiority of the Attune TKA concerning clinical outcome scores and ROM could be detected. This study adds to the literature as it represents the first, randomized, controlled trial to compare these two systems.

Studies have already been carried out to provide data following Attune and PFC TKA and this current study is in line with previous reports $[6,8,10,12]$. The new anatomical design was established to improve function and reduce knee pain, however, this could not yet be clearly observed. Ranawat et al. [10] found that in a 2-year follow-up matchedpair analysis no significant differences between the two groups in KS pain or function scores could be observed. The satisfaction rate for the Attune group was $97.9 \%$ and for the PFC group $96.6 \%$. According to the authors, modifications to the patellofemoral joint did not result in significant 
Table 2 Comparison of Clinical Outcome before and after 2-year follow-up

\begin{tabular}{lrrl}
\hline & Attune $(n=80)$ & PFC $(n=78)$ & $p$ value \\
\hline ROM $\left(^{\circ}\right)$ (mean \pm SD) & & & \\
Pre-operative & $93.4 \pm 21.8$ & $95.9 \pm 17.7$ & n.s \\
Post-operative & $113.0 \pm 10.6$ & $112.3 \pm 11.5$ & n.s \\
Change in ROM & $21.8 \pm 19.8$ & $21.5 \pm 15.6$ & n.s \\
WOMAC (mean \pm SD) & & & \\
Pre-operative & $53.7 \pm 14.0$ & $55.6 \pm 15.2$ & n.s \\
Post-operative & $86.7 \pm 15.3$ & $88.5 \pm 12.9$ & n.s \\
Change in WOMAC & $21.6 \pm 19.7$ & $18.1 \pm 12.0$ & n.s \\
KS (mean \pm SD) & & & \\
Pre-operative & $51.5 \pm 14.6$ & $52.3 \pm 13.6$ & n.s \\
Post-operative & $92.6 \pm 10.4$ & $88.8 \pm 14.3$ & n.s \\
Change in KSS pain & $42.0 \pm 16.8$ & $36.6 \pm 16.6$ & n.s \\
FS (mean \pm SD) & & & \\
Pre-operative & $44.6 \pm 12.2$ & $44.3 \pm 18.4$ & n.s \\
Post-operative & $85.0 \pm 19.3$ & $79.8 \pm 21.8$ & n.s \\
Change in KSS function & $41.8 \pm 21.7$ & $38.9 \pm 20.9$ & n.s \\
VAS (mean \pm SD) & & & \\
Pre-operative & $6.6 \pm 2.1$ & $6.5 \pm 1.9$ & n.s \\
Post-operative & $1.6 \pm 1.5$ & $1.6 \pm 1.8$ & n.s \\
Change in KSS function & $5.1 \pm 2.3$ & $5.0 \pm 2.1$ & n.s \\
\hline
\end{tabular}

Clinically and statistically, there were no significant (n.s.) differences in terms of pre- and postoperative ROM, WOMAC, KS, FS and VAS

improvements. Song et al. [12] showed more satisfactory results with the Attune TKA than the PFC regarding the KS, FS, WOMAC, and ROM after 2 years. In this study, a trend towards more favorable clinical results in the Attune group was detected with respect to ROM, KS and FS, however, without any statistical significance. Similar findings were also reported by Chua et al. [6], who reported that the newer TKA and the predecessor design achieved comparable improvements after 2 years. These authors even question the legitimacy of using a newer and costlier design in the absence of clear benefits. Another retrospective analysis by Molloy et al. [8] demonstrated that Attune implants do not appear to improve ROM and physical function outcomes relative to PFC.

The primary goal of the modification of a well-established implant should be an improvement of functional outcome and a decrease of unsatisfied patients. However, according to the present findings and aforementioned publications, theoretical advantages of the new design did not result in improvement of clinical outcome scores. It seems that the design might not be the sole key factor when it comes to functional outcome scores [2]. Other factors like the surgeon, the implantation technique, the surgical approach, the pain management and the patient characteristics might be just as important when determining the success of an implant. Behrend et al. [2] state that possibly only custommade implants can reproduce the anatomy sufficiently and that off-the-shelf prostheses might not offer room for further improvement [2]. Therefore, considering the usually higher costs of new developments, clinical and functional outcome scores are important indicators to prove economic reasonability before establishing and justifying the routine use of newer designs [6].

According to the local hospital database, all of the initial patients are free of revision surgery. However, it has recently been published that Attune prostheses show highly significant differences in the occurrence of radiolucent lines compared to PFC Sigma [13]. It will remain interesting if this occurrence leads to increased rates of aseptic loosening. Nonetheless, to date, there are no significant differences in terms of revision rates between the two prostheses in arthroplasty registers $[1,9]$.

There are several limitations to the study. First, we had a relatively high dropout rate, which could represent a selection bias. Although our study nurse contacted patients several times, most of them stated, that they were satisfied and therefore not willing to participate in the follow-up evaluation. Second, although we used validated clinical scores to evaluate postoperative pain and function, these clinical rating systems may not be sensitive enough to detect subtle changes. Therefore, a ceiling effect must be considered when interpreting the findings of this study. Third, our surgeons have already had a lot experience with the PFC Sigma, whereas especially in the initial study phase, the Attune TKA was relatively new. A higher skill level with respect to the PFC Sigma might have confounded the postoperative outcome. Despite these limitations, this first prospective, randomized, controlled study provides additional and valuable information on the comparison between a classic implant and its evolutional successor. Furthermore, the results emphasize the need for a prolonged follow-up evaluation.

\section{Conclusion}

The expected benefits of the Attune TKA modifications compared to its predecessor could not be observed in clinical outcome scores 2 year postoperatively. However, this study objectively demonstrated that both designs are effective options for improving pain and function following TKA. The results underscore the importance of qualitative research to clarify the performance of a traditional implant and its evolutional design. This information especially applies to surgeons when planning to change from a well-functioning implant, in which all characteristics and associated pitfalls are known, to a new, unknown design with missing practical experience. 
Acknowledgements Open access funding provided by Medical University of Graz.

Funding The authors received no financial support for the research, authorship and/or publication of this article.

\section{Compliance with ethical standards}

Conflict of interest Authors A.L. and P.S. have received institutional grants from Medtronic, Alphamed, Johnson\&Johnson not directly related to this study. The other authors declare that they have no conflict of interest.

Ethical approval The study has been performed in compliance with the Helsinki Declaration and has been approved by the local ethics committee (26-303 ex 13/14).

Open Access This article is licensed under a Creative Commons Attribution 4.0 International License, which permits use, sharing, adaptation, distribution and reproduction in any medium or format, as long as you give appropriate credit to the original author(s) and the source, provide a link to the Creative Commons licence, and indicate if changes were made. The images or other third party material in this article are included in the article's Creative Commons licence, unless indicated otherwise in a credit line to the material. If material is not included in the article's Creative Commons licence and your intended use is not permitted by statutory regulation or exceeds the permitted use, you will need to obtain permission directly from the copyright holder. To view a copy of this licence, visit http://creativecommons.org/licenses/by/4.0/.

\section{References}

1. Australian Orthopaedic Association National Joint Replacement Registry (2019) National Joint Replacement Registry. Annual Report 2019. Australian Orthopaedic Association National Joint Replacement Registry. https://aoanjrr.sahmri.com/annual-repor ts-2019

2. Behrend H, Zdravkovic V, Bosch M, Hochreiter B (2019) No difference in joint awareness after TKA: a matched-pair analysis of a classic implant and its evolutional design. Knee Surg Sports Traumatol Arthrosc 27:2124-2129

3. Bellamy N, Buchanan WW, Goldsmith CH, Campbell J, Stitt LW (1988) Validation study of WOMAC: a health status instrument for measuring clinically important patient relevant outcomes to antirheumatic drug therapy in patients with osteoarthritis of the hip or knee. J Rheumatol 15:1833-1840

4. Carey BW, Harty J (2018) A comparison of clinical- and patientreported outcomes of the cemented ATTUNE and PFC sigma fixed bearing cruciate sacrificing knee systems in patients who underwent total knee replacement with both prostheses in opposite knees. J Orthop Surg Res 13:54

5. Carr AJ, Robertsson O, Graves S, Price AJ, Arden NK, Judge A, Beard DJ (2012) Knee replacement. Lancet 379:1331-1340

6. Chua JL, Goh GS, Liow MHL, Tay DK, Lo NN, Yeo SJ (2019) Modern TKA implants are equivalent to traditional TKA implants in functional and patellofemoral joint-related outcomes. Knee Surg Sports Traumatol Arthrosc 27:1116-1123

7. Insall JN, Dorr LD, Scott RD, Scott WN (1989) Rationale of the Knee Society clinical rating system. Clin Orthop Relat Res 248:13-14

8. Molloy IB, Keeney BJ, Sparks MB, Paddock NG, Koenig KM, Moschetti WE, Jevsevar DS (2019) Short term patient outcomes after total knee arthroplasty: does the implant matter? Knee 26:687-699

9. National Joint Registry for England and Wales (2019) National Joint Registry. 16th Annual Report 2019. National Joint Registry for England and Wales. https://www.njrcentre.org.uk

10. Ranawat CS, White PB, West S, Ranawat AS (2017) Clinical and radiographic results of attune and PFC sigma knee designs at 2-year follow-up: a prospective matched-pair analysis. J Arthroplasty 32:431-436

11. Sadoghi P, Kastner N (2013) Size measurement and flexion gap balancing in total knee arthroplasty-new benefits of the attune system? Int Orthop 37:2105

12. Song SJ, Kang SG, Park CH, Bae DK (2018) Comparison of clinical results and risk of patellar injury between attune and PFC sigma knee systems. Knee Surg Relat Res 30:334-340

13. Staats K, Wannmacher T, Weihs V, Koller U, Kubista B, Windhager R (2019) Modern cemented total knee arthroplasty design shows a higher incidence of radiolucent lines compared to its predecessor. Knee Surg Sports Traumatol Arthrosc 27:1148-1155

Publisher's Note Springer Nature remains neutral with regard to jurisdictional claims in published maps and institutional affiliations. 